\title{
Extremal Solutions and Relaxation Problems for Fractional Differential Inclusions
}

\author{
Juan J. Nieto, ${ }^{1,2}$ Abdelghani Ouahab, ${ }^{3}$ and P. Prakash ${ }^{1,4}$ \\ ${ }^{1}$ Departamento de Análisis Matematico, Facultad de Matemáticas, Universidad de Santiago de Compostela, \\ 15782 Santiago de Compostela, Spain \\ ${ }^{2}$ Department of Mathematics, King Abdulaziz University, Jeddah 21589, Saudi Arabia \\ ${ }^{3}$ Laboratory of Mathematics, Sidi-Bel-Abbès University, P.O. Box 89, 22000 Sidi-Bel-Abbès, Algeria \\ ${ }^{4}$ Department of Mathematics, Periyar University, Salem 636 011, India
}

Correspondence should be addressed to Juan J. Nieto; juanjose.nieto.roig@usc.es

Received 10 May 2013; Accepted 31 July 2013

Academic Editor: Daniel C. Biles

Copyright (C) 2013 Juan J. Nieto et al. This is an open access article distributed under the Creative Commons Attribution License, which permits unrestricted use, distribution, and reproduction in any medium, provided the original work is properly cited.

We present the existence of extremal solution and relaxation problem for fractional differential inclusion with initial conditions.

\section{Introduction}

Differential equations with fractional order have recently proved to be valuable tools in the modeling of many physical phenomena [1-9]. There has also been a significant theoretical development in fractional differential equations in recent years; see the monographs of Kilbas et al. [10], Miller and Ross [11], Podlubny [12], and Samko et al. [13] and the papers of Kilbas and Trujillo [14], Nahušev [15], Podlubny et al. [16], and $\mathrm{Yu}$ and Gao [17].

Recently, some basic theory for initial value problems for fractional differential equations and inclusions involving the Riemann-Liouville differential operator was discussed, for example, by Lakshmikantham [18] and Chalco-Cano et al. [19].

Applied problems requiring definitions of fractional derivatives are those that are physically interpretable for initial conditions containing $y(0), y^{\prime}(0)$, and so forth. The same requirements are true for boundary conditions. Caputo's fractional derivative satisfies these demands. For more details on the geometric and physical interpretation for fractional derivatives of both Riemann-Liouville and Caputo types, see Podlubny [12].

Fractional calculus has a long history. We refer the reader to $[20]$.

Recently fractional functional differential equations and inclusions and impulsive fractional differential equations and inclusions with standard Riemann-Liouville and Caputo derivatives with differences conditions were studied by Abbas et al. [21, 22], Benchohra et al. [23], Henderson and Ouahab [24, 25], Jiao and Zhou [26], and Ouahab [27-29] and in the references therein.

In this paper, we will be concerned with the existence of solutions, Filippov's theorem, and the relaxation theorem of abstract fractional differential inclusions. More precisely, we will consider the following problem:

$$
\begin{gathered}
{ }^{c} D^{\alpha} y(t) \in F(t, y(t)), \quad \text { a.e. } t \in J:=[0, b], \\
y(0)=y_{0}, \quad y^{\prime}(0)=y_{1}, \\
{ }^{c} D^{\alpha} y(t) \in \operatorname{ext} F(t, y(t)), \quad \text { a.e. } t \in J:=[0, b], \\
y(0)=y_{0}, \quad y^{\prime}(0)=y_{1},
\end{gathered}
$$

where ${ }^{c} D^{\alpha}$ is the Caputo fractional derivatives, $\alpha \in(1,2]$, $F: J \times \mathbb{R}^{N} \rightarrow \mathscr{P}\left(\mathbb{R}^{N}\right)$ is a multifunction, and ext $F(t, y)$ represents the set of extreme points of $F(t, y) .\left(\mathscr{P}\left(\mathbb{R}^{N}\right)\right.$ is the family of all nonempty subsets of $\mathbb{R}^{N}$.

During the last couple of years, the existence of extremal solutions and relaxation problem for ordinary differential inclusions was studied by many authors, for example, see [30$34]$ and the references therein. 
The paper is organized as follows. We first collect some background material and basic results from multivalued analysis and give some results on fractional calculus in Sections 2 and 3, respectively. Then, we will be concerned with the existence of solution for extremal problem. This is the aim of Section 4. In Section 5, we prove the relaxation problem.

\section{Preliminaries}

The reader is assumed to be familiar with the theory of multivalued analysis and differential inclusions in Banach spaces, as presented in Aubin et al. [35, 36], Hu and Papageorgiou [37], Kisielewicz [38], and Tolstonogov [32].

Let $(X,\|\cdot\|)$ be a real Banach space, $[0, b]$ an interval in $R$, and $C([0, b], X)$ the Banach space of all continuous functions from $J$ into $X$ with the norm

$$
\|y\|_{\infty}=\sup \{\|y(t)\|: 0 \leq t \leq b\} .
$$

A measurable function $y:[0, b] \rightarrow X$ is Bochner integrable if $\|y\|$ is Lebesgue integrable. In what follows, $L^{1}([0, b], X)$ denotes the Banach space of functions $y$ : $[0, b] \rightarrow X$, which are Bochner integrable with norm

$$
\|y\|_{1}=\int_{0}^{b}\|y(t)\| d t
$$

Denote by $L_{w}^{1}([0, b], X)$ the space of equivalence classes of Bochner integrable function $y:[0, b] \rightarrow X$ with the norm

$$
\|y\|_{w}=\sup _{t \in[0, t]}\left\|\int_{0}^{t} y(s) d s\right\| .
$$

The norm $\|\cdot\|_{w}$ is weaker than the usual norm $\|\cdot\|_{1}$, and for a broad class of subsets of $L^{1}([0, b], X)$, the topology defined by the weak norm coincides with the usual weak topology (see [37, Proposition 4.14, page 195]). Denote by

$$
\begin{gathered}
\mathscr{P}(X)=\{Y \subset X: Y \neq \emptyset\}, \\
\mathscr{P}_{\mathrm{cl}}(X)=\{Y \in \mathscr{P}(X): Y \text { closed }\}, \\
\mathscr{P}_{b}(X)=\{Y \in \mathscr{P}(X): Y \text { bounded }\}, \\
\mathscr{P}_{\mathrm{cv}}(X)=\{Y \in \mathscr{P}(X): Y \text { convex }\}, \\
\mathscr{P}_{\mathrm{cp}}(X)=\{Y \in \mathscr{P}(X): Y \text { compact }\} .
\end{gathered}
$$

A multivalued map $G: X \rightarrow \mathscr{P}(X)$ has convex (closed) values if $G(x)$ is convex (closed) for all $x \in X$. We say that $G$ is bounded on bounded sets if $G(B)$ is bounded in $X$ for each bounded set $B$ of $X$ (i.e., $\sup _{x \in B}\{\sup \{\|y\|: y \in G(x)\}\}<\infty$ ).

Definition 1. A multifunction $F: X \rightarrow \mathscr{P}(Y)$ is said to be upper semicontinuous at the point $x_{0} \in X$, if, for every open $W \subseteq Y$ such that $F\left(x_{0}\right) \subset W$, there exists a neighborhood $V\left(x_{0}\right)$ of $x_{0}$ such that $F\left(V\left(x_{0}\right)\right) \subset W$.

A multifunction is called upper semicontinuous (u.s.c. for short) on $X$ if for each $x \in X$ it is u.s.c. at $x$.
Definition 2. A multifunction $F: X \rightarrow \mathscr{P}(Y)$ is said to be lower continuous at the point $x_{0} \in X$, if, for every open $W \subseteq$ $Y$ such that $F\left(x_{0}\right) \cap W \neq \emptyset$, there exists a neighborhood $V\left(x_{0}\right)$ of $x_{0}$ with property that $F(x) \cap W \neq \emptyset$ for all $x \in V\left(x_{0}\right)$.

A multifunction is called lower semicontinuous (l.s.c. for short) provided that it is lower semicontinuous at every point $x \in X$.

Lemma 3 (see [39, Lemma 3.2]). Let $F:[0, b] \rightarrow \mathscr{P}(Y)$ be a measurable multivalued map and $u:[a, b] \rightarrow Y a$ measurable function. Then for any measurable $v:[a, b] \rightarrow$ $(0,+\infty)$, there exists a measurable selection $f_{v}$ of $F$ such that for a.e. $t \in[a, b]$,

$$
\left\|u(t)-f_{v}(t)\right\| \leq d(u(t), F(t))+v(t) .
$$

First, consider the Hausdorff pseudometric

$$
H_{d}: \mathscr{P}(E) \times \mathscr{P}(E) \longrightarrow \mathbb{R}^{+} \cup\{\infty\}
$$

defined by

$$
H_{d}(A, B)=\max \left\{\sup _{a \in A} d(a, B), \sup _{b \in B} d(A, b)\right\},
$$

where $d(A, b)=\inf _{a \in A} d(a, b)$ and $d(a, B)=\inf _{b \in B} d(a, b)$. $\left(\mathscr{P}_{b, \mathrm{cl}}(E), H_{d}\right)$ is a metric space and $\left(\mathscr{P}_{\mathrm{cl}}(X), H_{d}\right)$ is a generalized metric space.

Definition 4. A multifunction $F: Y \rightarrow \mathscr{P}(X)$ is called Hausdorff lower semicontinuous at the point $y_{0} \in Y$, if for any $\epsilon>0$ there exists a neighbourhood $U\left(y_{0}\right)$ of the point $y_{0}$ such that

$$
F\left(y_{0}\right) \subset F(y)+\epsilon B(0,1), \quad \text { for every } y \in U\left(y_{0}\right) \text {, }
$$

where $B(0,1)$ is the unite ball in $X$.

Definition 5. A multifunction $F: Y \rightarrow \mathscr{P}(X)$ is called Hausdorff upper semicontinuous at the point $y_{0} \in Y$, if for any $\epsilon>0$ there exists a neighbourhood $U\left(y_{0}\right)$ of the point $y_{0}$ such that

$$
F(y) \subset F\left(y_{0}\right)+\epsilon B(0,1), \quad \text { for every } y \in U\left(y_{0}\right) \text {. }
$$

$F$ is called continuous, if it is Hausdorff lower and upper semicontinuous.

Definition 6. Let $X$ be a Banach space; a subset $A \subset$ $L^{1}([0, b], X)$ is decomposable if, for all $u, v \in A$ and for every Lebesgue measurable set $I \subset J$, one has

$$
u \chi_{I}+v \chi_{[0, b] \backslash I} \in A
$$

where $\chi_{A}$ stands for the characteristic function of the set $A$. We denote by $\operatorname{Dco}\left(L^{1}([0, b], X)\right)$ the family of decomposable sets. 
Let $F:[0, b] \times X \rightarrow \mathscr{P}(X)$ be a multivalued map with nonempty closed values. Assign to $F$ the multivalued operator $\mathscr{F}: C([0, b], X) \rightarrow \mathscr{P}\left(L^{1}([0, b], X)\right)$ defined by

$$
\begin{aligned}
\mathscr{F}(y)=\left\{v \in L^{1}([0, b], X): v(t) \in F(t, y(t)),\right. \\
\text { a.e. } t \in[0, b]\} .
\end{aligned}
$$

The operator $\mathscr{F}$ is called the Nemyts'kii operator associated to $F$.

Definition 7. Let $F:[0, b] \times X \rightarrow \mathscr{P}(X)$ be a multivalued map with nonempty compact values. We say that $F$ is of lower semicontinuous type (l.s.c. type) if its associated Nemyts'kil operator $\mathscr{F}$ is lower semicontinuous and has nonempty closed and decomposable values.

Next, we state a classical selection theorem due to Bressan and Colombo.

Lemma 8 (see [40]). Let $X$ be a separable metric space and let $E$ be a Banach space. Then every l.s.c. multivalued operator $N$ : $X \rightarrow \mathscr{P}_{c l}\left(L^{1}([0, b], E)\right)$ with closed decomposable values has a continuous selection; that is, there exists a continuous singlevalued function $f: X \rightarrow L^{1}([0, b], E)$ such that $f(x) \in N(x)$ for every $x \in X$.

Let us introduce the following hypothesis.

$\left(\overline{\mathscr{H}}_{1}\right) F:[0, b] \times X \rightarrow \mathscr{P}(X)$ is a nonempty compact valued multivalued map such that

(a) the mapping $(t, y) \mapsto F(t, y)$ is $\mathscr{L} \otimes \mathscr{B}$ measurable;

(b) the mapping $y \mapsto F(t, y)$ is lower semicontinuous for a.e. $t \in[0, b]$.

Lemma 9 (see, e.g., [41]). Let $F: J \times X \rightarrow \mathscr{P}_{c p}(E)$ be an integrably bounded multivalued map satisfying $\left(\overline{\mathscr{H}}_{1}\right)$. Then $F$ is of lower semicontinuous type.

Define

$$
\begin{array}{r}
F(K)=\left\{f \in L^{1}([0, b], X): f(t) \in K \text { a.e. } t \in[0, b]\right\}, \\
K \subset X,
\end{array}
$$

where $X$ is a Banach space.

Lemma 10 (see [37]). Let $K \subset X$ be a weakly compact subset of $X$. Then $F(K)$ is relatively weakly compact subset of $L^{1}([0, b], X)$. Moreover if $K$ is convex, then $F(K)$ is weakly compact in $L^{1}([0, b], X)$.

Definition 11. A multifunction $F:[0, b] \times Y \rightarrow \mathscr{P}_{\text {wcpcv }}(X)$ possesses the Scorza-Dragoni property (S-D property) if for each $\epsilon>0$, there exists a closed set $J_{\epsilon} \subset[0, b]$ whose Lebesgue measure $\mu\left(J_{\epsilon}\right) \leq \epsilon$ and such that $F:[0, b] \backslash J_{\epsilon} \times Y \rightarrow X$ is continuous with respect to the metric $d_{X}(\cdot, \cdot)$.
Remark 12. It is well known that if the map $F:[0, b] \times Y \rightarrow$ $\mathscr{P}_{\text {wcpcv }}(X)$ is continuous with respect to $y$ for almost every $t \in[0, b]$ and is measurable with respect to $t$ for every $y \in Y$, then it possesses the $\mathrm{S}-\mathrm{D}$ property.

In what follows, we present some definitions and properties of extreme points.

Definition 13. Let $A$ be a nonempty subset of a real or complex linear vector space. An extreme point of a convex set $A$ is a point $x \in A$ with the property that if $x=\lambda y+(1-\lambda) z$ with $y, z \in A$ and $\lambda \in[0,1]$, then $y=x$ and/or $z=x$. $\operatorname{ext}(A)$ will denote the set of extreme points of $A$.

In other words, an extreme point is a point that is not an interior point of any line segment lying entirely in $A$.

Lemma 14 (see [42]). A nonempty compact set in a locally convex linear topological space has extremal points.

Let $\left\{x_{n}^{\prime}\right\}_{n \in \mathbb{N}}$ be a denumerable, dense (in $\sigma\left(X^{\prime}, X\right)$ topology) subset of the set $\{x \in X:\|x\| \leq 1\}$. For any $A \in$ $\mathscr{P}_{\text {cpcv }}(X)$ and $x_{n}^{\prime}$ define the function

$$
d^{n}(A, u)=\max \left\{\left\langle y-z, x_{n}^{\prime}\right\rangle: y, z \in A, u=\frac{y+z}{2}\right\} .
$$

Lemma 15 (see [33]). $u \in \operatorname{ext}(A)$ if and only if $d^{n}(A, u)=0$ for all $n \geq 1$.

In accordance with Krein-Milman and Trojansky theorem [43], the set $\operatorname{ext}\left(S_{F}\right)$ is nonempty and $\overline{\operatorname{co}}\left(\operatorname{ext}\left(S_{F}\right)\right)=S_{F}$.

Lemma 16 (see [33]). Let $F:[0, b] \rightarrow \mathscr{P}_{w c p c v}(X)$ be a measurable, integrably bounded map. Then

$$
\overline{\operatorname{ext}}\left(S_{F}\right) \subseteq S_{F},
$$

where $\overline{\operatorname{ext}}\left(S_{F}\right)$ is the closure of set ext $\left(S_{F}\right)$ in the topology of the space $L^{1}([0, b], X)$.

Theorem 17 (see [33]). Let $F:[0, b] \times Y \rightarrow \mathscr{P}_{w c p c v}(X)$ be a multivalued map that has the S-D property and let it be integrable bounded on compacts from $Y$. Consider a compact subset $K \subset C([0, b], X)$ and define the multivalued map $G$ : $K \rightarrow L^{1}([0, b], X), b y$

$$
\begin{array}{r}
G(y(\cdot)) \\
=\left\{f \in L^{1}([0, b], X): f(t) \in F(t, y(t)) \text { a.e. on }[0, b]\right\}, \\
y \in K .
\end{array}
$$

Then for every $K$ compact in $C([0, b], X), \epsilon>0$ and any continuous selection $f: K \rightarrow L^{1}([0, b], X)$, there exists a continuous selector $g: K \rightarrow L^{1}([0, b], X)$ of the map ext $(G)$ : $K \rightarrow L^{1}([0, b], X)$ such that for all $y \in C([0, b], X)$ one has

$$
\sup _{t \in[0, b]}\left\|\int_{0}^{t}((f y)(s)-(g y)(s)) d s\right\| \leq \epsilon .
$$


For a background of extreme point of $F(t, y(t))$ see Dunford-Schwartz [42, Chapter 5, Section 8] and Florenzano and Le Van [44, Chapter 3].

\section{Fractional Calculus}

According to the Riemann-Liouville approach to fractional calculus, the notation of fractional integral of order $\alpha(\alpha>0)$ is a natural consequence of the well known formula (usually attributed to Cauchy) that reduces the calculation of the $n$-fold primitive of a function $f(t)$ to a single integral of convolution type. In our notation the Cauchy formula reads

$$
I^{n} f(t):=\frac{1}{(n-1) !} \int_{0}^{t}(t-s)^{n-1} f(s) d s, \quad t>0, n \in \mathbb{N} \text {. }
$$

Definition 18 (see $[13,45]$ ). The fractional integral of order $\alpha>0$ of a function $f \in L^{1}([a, b], \mathbb{R})$ is defined by

$$
I_{a^{+}}^{\alpha} f(t)=\int_{a}^{t} \frac{(t-s)^{\alpha-1}}{\Gamma(\alpha)} f(s) d s
$$

where $\Gamma$ is the gamma function. When $a=0$, we write $I^{\alpha} f(t)=f(t) * \phi_{\alpha}(t)$, where $\phi_{\alpha}(t)=t^{(\alpha-1)} / \Gamma(\alpha)$ for $t>0$, and we write $\phi_{\alpha}(t)=0$ for $t \leq 0$ and $\phi_{\alpha} \rightarrow \delta(t)$ as $\alpha \rightarrow 0$, where $\delta$ is the delta function and $\Gamma$ is the Euler gamma function defined by

$$
\Gamma(\alpha)=\int_{0}^{\infty} t^{\alpha-1} e^{-t} d t, \quad \alpha>0
$$

For consistency, $I^{0}=$ Id (identity operator), that is, $I^{0} f(t)=$ $f(t)$. Furthermore, by $I^{\alpha} f\left(0^{+}\right)$we mean the limit (if it exists) of $I^{\alpha} f(t)$ for $t \rightarrow 0^{+}$; this limit may be infinite.

After the notion of fractional integral, that of fractional derivative of order $\alpha(\alpha>0)$ becomes a natural requirement and one is attempted to substitute $\alpha$ with $-\alpha$ in the above formulas. However, this generalization needs some care in order to guarantee the convergence of the integral and preserve the well known properties of the ordinary derivative of integer order. Denoting by $D^{n}$, with $n \in \mathbb{N}$, the operator of the derivative of order $n$, we first note that

$$
D^{n} I^{n}=\mathrm{Id}, \quad I^{n} D^{n} \neq \mathrm{Id}, \quad n \in \mathbb{N},
$$

that is, $D^{n}$ is the left inverse (and not the right inverse) to the corresponding integral operator $J^{n}$. We can easily prove that

$$
I^{n} D^{n} f(t)=f(t)-\sum_{k=0}^{n-1} f^{(k)}\left(a^{+}\right) \frac{(t-a)^{k}}{k !}, \quad t>0 .
$$

As a consequence, we expect that $D^{\alpha}$ is defined as the left inverse to $I^{\alpha}$. For this purpose, introducing the positive integer $n$ such that $n-1<\alpha \leq n$, one defines the fractional derivative of order $\alpha>0$.
Definition 19. For a function $f$ given on interval $[a, b]$, the $\alpha$ th Riemann-Liouville fractional-order derivative of $f$ is defined by

$$
D^{\alpha} f(t)=\frac{1}{\Gamma(n-\alpha)}\left(\frac{d}{d t}\right)^{n} \int_{a}^{t}(t-s)^{-\alpha+n-1} f(s) d s
$$

where $n=[\alpha]+1$ and $[\alpha]$ is the integer part of $\alpha$.

Defining for consistency, $D^{0}=I^{0}=\mathrm{Id}$, then we easily recognize that

$$
\begin{gathered}
D^{\alpha} I^{\alpha}=\mathrm{Id}, \quad \alpha \geq 0, \\
D^{\alpha} t^{\gamma}=\frac{\Gamma(\gamma+1)}{\Gamma(\gamma+1-\alpha)} t^{\gamma-\alpha}, \\
\alpha>0, \quad \gamma \in(-1,0) \cup(0,+\infty), \quad t>0 .
\end{gathered}
$$

Of course, properties (25) and (26) are a natural generalization of those known when the order is a positive integer.

Note the remarkable fact that the fractional derivative $D^{\alpha} f$ is not zero for the constant function $f(t)=1$, if $\alpha \notin \mathbb{N}$. In fact, (26) with $\gamma=0$ illustrates that

$$
D^{\alpha} 1=\frac{(t-a)^{-\alpha}}{\Gamma(1-\alpha)}, \quad \alpha>0, t>0 .
$$

It is clear that $D^{\alpha} 1=0$, for $\alpha \in \mathbb{N}$, due to the poles of the gamma function at the points $0,-1,-2, \ldots$.

We now observe an alternative definition of fractional derivative, originally introduced by Caputo $[46,47]$ in the late sixties and adopted by Caputo and Mainardi [48] in the framework of the theory of Linear Viscoelasticity (see a review in [4]).

Definition 20. Let $f \in A C^{n}([a, b])$. The Caputo fractionalorder derivative of $f$ is defined by

$$
\left({ }^{c} D^{\alpha} f\right)(t):=\frac{1}{\Gamma(n-\alpha)} \int_{a}^{t}(t-s)^{n-\alpha-1} f^{n}(s) d s .
$$

This definition is of course more restrictive than Riemann-Liouville definition, in that it requires the absolute integrability of the derivative of order $m$. Whenever we use the operator $D_{*}^{\alpha}$ we (tacitly) assume that this condition is met. We easily recognize that in general

$$
D^{\alpha} f(t):=D^{m} I^{m-\alpha} f(t) \neq J^{m-\alpha} D^{m} f(t):=D_{*}^{\alpha} f(t),
$$

unless the function $f(t)$, along with its first $n-1$ derivatives, vanishes at $t=a^{+}$. In fact, assuming that the passage of the $m$ derivative under the integral is legitimate, we recognize that, for $m-1<\alpha<m$ and $t>0$,

$$
D^{\alpha} f(t)={ }^{c} D^{\alpha} f(t)+\sum_{k=0}^{m-1} \frac{(t-a)^{k-\alpha}}{\Gamma(k-\alpha+1)} f^{(k)}\left(a^{+}\right),
$$

and therefore, recalling the fractional derivative of the power function (26), one has

$$
D^{\alpha}\left(f(t)-\sum_{k=0}^{m-1} \frac{(t-a)^{k-\alpha}}{\Gamma(k-\alpha+1)} f^{(k)}\left(a^{+}\right)\right)=D_{*}^{\alpha} f(t) .
$$


The alternative definition, that is, Definition 20, for the fractional derivative thus incorporates the initial values of the function and of lower order. The subtraction of the Taylor polynomial of degree $n-1$ at $t=a^{+}$from $f(t)$ means a sort of regularization of the fractional derivative. In particular, according to this definition, the relevant property for which the fractional derivative of a constant is still zero:

$$
{ }^{c} D^{\alpha} 1=0, \quad \alpha>0 .
$$

We now explore the most relevant differences between the two fractional derivatives given in Definitions 19 and 20. From Riemann-Liouville fractional derivatives, we have

$$
D^{\alpha}(t-a)^{\alpha-j}=0, \quad \text { for } j=1,2, \ldots,[\alpha]+1 .
$$

From (32) and (33) we thus recognize the following statements about functions which, for $t>0$, admit the same fractional derivative of $\operatorname{order} \alpha$, with $n-1<\alpha \leq n, n \in \mathbb{N}$ :

$$
\begin{gathered}
D^{\alpha} f(t)=D^{\alpha} g(t) \Longleftrightarrow f(t)=g(t)+\sum_{j=1}^{m} c_{j}(t-a)^{\alpha-j}, \\
{ }^{c} D^{\alpha} f(t)={ }^{c} D^{\alpha} g(t) \Longleftrightarrow f(t)=g(t)+\sum_{j=1}^{m} c_{j}(t-a)^{n-j} .
\end{gathered}
$$

In these formulas, the coefficients $c_{j}$ are arbitrary constants. For proving all main results we present the following auxiliary lemmas.

Lemma 21 (see [10]). Let $\alpha>0$ and let $y \in L^{\infty}(a, b)$ or $C([a, b])$. Then

$$
\left({ }^{c} D^{\alpha} I^{\alpha} y\right)(t)=y(t)
$$

Lemma 22 (see [10]). Let $\alpha>0$ and $n=[\alpha]+1$. If $y \in$ $A C^{n}[a, b]$ or $y \in C^{n}[a, b]$, then

$$
\left(I^{\alpha c} D^{\alpha} y\right)(t)=y(t)-\sum_{k=0}^{n-1} \frac{y^{(k)}(a)}{k !}(t-a)^{k} .
$$

For further readings and details on fractional calculus, we refer to the books and papers by Kilbas [10], Podlubny [12], Samko [13], and Caputo [46-48].

\section{Existence Result}

Definition 23. A function $y \in C\left([0, b], \mathbb{R}^{N}\right)$ is called mild solution of problem (1) if there exist $f \in L^{1}\left(J, \mathbb{R}^{N}\right)$ such that

$$
y(t)=y_{0}+t y_{1}+\frac{1}{\Gamma(\alpha)} \int_{0}^{t}(t-s)^{1-\alpha} f(s) d s, \quad t \in[0, b],
$$

where $f \in S_{F, y}=\left\{v \in L^{1}\left([0, b], \mathbb{R}^{N}\right): f(t) \in F(t, y(t))\right.$ a.e. on $[0, b]\}$.

We will impose the following conditions on $F$.
$\left(\mathscr{H}_{1}\right)$ The function $F: J \times \mathbb{R}^{N} \rightarrow \mathscr{P}_{\text {cpcv }}\left(\mathbb{R}^{N}\right)$ such that

(a) for all $x \in \mathbb{R}^{N}$, the map $t \mapsto F(t, x)$ is measurable,

(b) for every $t \in[0, b]$, the multivalued map $x \rightarrow$ $F(t, x)$ is $H_{d}$ continuous

$\left(\mathscr{H}_{2}\right)$ There exist $p \in L^{1}\left(J, \mathbb{R}^{+}\right)$and a continuous nondecreasing function $\psi:[0, \infty) \rightarrow(0, \infty)$ such that

$$
\begin{array}{r}
\|F(t, x)\|_{\mathscr{D}}=\sup \{\|v\|: v \in F(t, x)\} \leq p(t) \psi(\|x\|), \\
\text { for a.e. } t \in[0, b] \text { and each } x \in \mathbb{R}^{N},
\end{array}
$$

with

$$
\int_{0}^{b} p(s) d s<\int_{\left\|y_{0}\right\|+b\left\|y_{1}\right\|}^{\infty} \frac{d u}{\psi(u)}
$$

Theorem 24. Assume that the conditions $\left(\mathscr{H}_{1}\right)-\left(\mathscr{H}_{2}\right)$ and then the problem (2) have at least one solution.

Proof. From $\left(\mathscr{H}_{2}\right)$ there exists $M>0$ such that $\|y\|_{\infty} \leq M$ for each $y \in S_{c}$.

Let

$$
F_{1}(t, y)= \begin{cases}F(t, y) & \text { if }\|y\| \leq M, \\ F\left(t, \frac{M y}{\|y\|}\right) & \text { if }\|y\| \geq M .\end{cases}
$$

We consider

$$
\begin{gathered}
{ }^{c} D^{\alpha} y(t) \in F_{1}(t, y(t)), \quad \text { a.e. } t \in[0, b], \\
y(0)=y_{0}, \quad y^{\prime}(0)=y_{1} .
\end{gathered}
$$

It is clear that all the solutions of (41) are solutions of (2).

Set

$$
\begin{array}{r}
V=\left\{f \in L^{1}\left([0, b], \mathbb{R}^{N}\right):\|f(t)\| \leq \psi_{*}(t)\right\}, \\
\psi_{*}(t)=p(t) \psi(M) .
\end{array}
$$

It is clear that $V$ is weakly compact in $L^{1}\left([0, b], \mathbb{R}^{N}\right)$. Remark that for every $f \in V$, there exists a unique solution $L(f)$ of the following problem:

$$
\begin{aligned}
& { }^{c} D^{\alpha} y(t)=f(t), \quad \text { a.e. } t \in[0, b], \\
& y(t)=y_{0}, \quad y^{\prime}(0)=y_{1} ;
\end{aligned}
$$

this solution is defined by

$$
L(f)(t)=y_{0}+t y_{1}+\frac{1}{\Gamma(\alpha)} \int_{0}^{t}(t-s)^{\alpha-1} f(s) d s,
$$

$$
\text { a.e. } t \in[0, b] \text {. }
$$

We claim that $L$ is continuous. Indeed, let $f_{n} \rightarrow f$ converge in $L^{1}\left([0, b], \mathbb{R}^{N}\right)$, as $n \rightarrow \infty$, set $y_{n}=L\left(f_{n}\right), n \in \mathbb{N}$. It is clear 
that $\left\{y_{n}: n \in \mathbb{N}\right\}$ is relatively compact in $C\left([0, b], \mathbb{R}^{N}\right)$ and $y_{n}$ converge to $y \in C\left([0, b], \mathbb{R}^{N}\right)$. Let

$$
z(t)=y_{0}+y_{1} t+\frac{1}{\Gamma(\alpha)} \int_{0}^{t}(t-s)^{\alpha-1} f(s) d s, \quad t \in[0, b]
$$

Then

$$
\begin{array}{r}
\left\|y_{n}-z\right\|_{\infty} \leq \frac{b^{\alpha}}{\Gamma(\alpha)} \int_{0}^{b}\left\|f_{n}(s)-f(s)\right\| d s \\
\text { as } n \longrightarrow 0,
\end{array}
$$

Hence $K=L(V)$ is compact and convex subset of $C\left([0, b], \mathbb{R}^{N}\right)$. Let $S_{F}: K \rightarrow \mathscr{P}_{\text {clcv }}\left(L^{1}\left([0, b], \mathbb{R}^{N}\right)\right)$ be the multivalued Nemitsky operator defined by

$$
\begin{gathered}
S_{F_{1}}(y)=\left\{f \in L^{1}\left([0, b], \mathbb{R}^{N}\right): f(t) \in F_{1}(t, y(t)),\right. \\
\text { a.e. } t \in[0, b]\}:=S_{F_{1}, y} .
\end{gathered}
$$

It is clear that $F_{1}(\cdot, \cdot)$ is $H_{d}$ continuous and $F_{1}(\cdot, \cdot) \quad \epsilon$ $\mathscr{P}_{w k c p c v}\left(\mathbb{R}^{N}\right)$ and is integrably bounded, then by Theorem 17 (see also Theorem 6.5 in [32] or Theorem 1.1 in [34]), we can find a continuous function $g: K \rightarrow L_{w}^{1}\left([0, b], \mathbb{R}^{N}\right)$ such that

$$
g(x) \in \operatorname{ext} S_{F_{1}}(y) \quad \forall y \in K
$$

From Benamara [49] we know that

$$
\operatorname{ext} S_{F_{1}}(y)=S_{\text {ext } F_{1}(\cdot, y(\cdot))} \quad \forall y \in K \text {. }
$$

Setting $N=L \circ g$ and letting $y \in K$, then

$$
\begin{aligned}
g(y) & \in F_{1}(\cdot, y(\cdot)) \Longrightarrow g(y) \in V \Longrightarrow N(y) \\
& =L(g(y)) \in K .
\end{aligned}
$$

Now, we prove that $N$ is continuous. Indeed, let $y_{n} \in K$ converge to $y$ in $C\left([0, b], \mathbb{R}^{N}\right)$.

Then

$$
g\left(y_{n}\right) \text { converge weakly to } g(y) \text { as } n \longrightarrow \infty \text {. }
$$

Since $N\left(y_{n}\right)=L\left(g\left(y_{n}\right)\right) \in K$ and $g\left(y_{n}\right)(\cdot) \in F\left(t, y_{n}(t)\right)$, then

$$
g\left(y_{n}\right)(\cdot) \in F\left(\cdot, \bar{B}_{M}\right) \in \mathscr{P}_{\mathrm{cp}}\left(\mathbb{R}^{N}\right)
$$

From Lemma $10, g\left(y_{n}\right)$ converge weakly to $y$ in $L^{1}\left([0, b], \mathbb{R}^{N}\right)$ as $n \rightarrow \infty$. By the definition of $N$, we have

$$
\begin{array}{r}
N\left(y_{n}\right)=y_{0}+y_{1} t+\frac{1}{\Gamma(\alpha)} \int_{0}^{t}(t-s)^{\alpha-1} g\left(y_{n}\right)(s) d s \\
t \in[0, b], \\
N(y)=y_{0}+y_{1} t+\frac{1}{\Gamma(\alpha)} \int_{0}^{t}(t-s)^{\alpha-1} g(y)(s) d s,
\end{array}
$$

Since $\left\{N\left(y_{n}\right): n \in \mathbb{N}\right\} \subset K$, then there exists subsequence of $N\left(y_{n}\right)$ converge in $C\left([0, b], \mathbb{R}^{N}\right)$. Then

$$
N\left(y_{n}\right)(t) \longrightarrow N(y)(t), \quad \forall t \in[0, b], \text { as } n \longrightarrow \infty .
$$

This proves that $N$ is continuous. Hence by Schauder's fixed point there exists $y \in K$ such that $y=N(y)$.

\section{The Relaxed Problem}

In this section, we examine whether the solutions of the extremal problem are dense in those of the convexified one. Such a result is important in optimal control theory whether the relaxed optimal state can be approximated by original states; the relaxed problems are generally much simpler to build. For the problem for first-order differential inclusions, we refer, for example, to [35, Theorem 2, page 124] or [36, Theorem 10.4.4, page 402]. For the relaxation of extremal problems we see the following recent references $[30,50]$.

Now we present our main result of this section.

Theorem 25. Let $F:[0, b] \times \mathbb{R}^{N} \rightarrow \mathscr{P}\left(\mathbb{R}^{N}\right)$ be a multifunction satisfying the following hypotheses.

$\left(\mathscr{H}_{3}\right)$ The function $F:[0, b] \times \mathbb{R}^{N} \rightarrow \mathscr{P}_{c p c v}\left(\mathbb{R}^{N}\right)$ such that, for all $x \in \mathbb{R}^{N}$, the map

$$
t \longmapsto F(t, x)
$$

is measurable.

$\left(\mathscr{H}_{4}\right)$ There exists $p \in L^{1}\left(J, \mathbb{R}^{+}\right)$such that

$$
\begin{gathered}
H_{d}(F(t, x), F(t, y)) \leq p(t)\|x-y\|, \\
\text { for a.e. } t \in[0, b] \text { and each } x, y \in \mathbb{R}^{N}, \\
H_{d}(F(t, 0), 0) \leq p(t) \quad \text { for a.e. } t \in[0, b] .
\end{gathered}
$$

Then $\overline{S_{e}}=S_{c}$.

Proof. By Coviz and Nadlar fixed point theorem, we can easily prove that $S_{c} \neq \emptyset$, and since $F$ has compact and convex valued, then $S_{c}$ is compact in $C\left([0, b], \mathbb{R}^{N}\right)$. For more information we see $[25,27-29,51,52]$.

Let $y \in S_{c}$; then there exists $f \in S_{F, y}$ such that

$$
\begin{array}{r}
y(t)=y_{0}+y_{1} t+\frac{1}{\Gamma(\alpha)} \int_{0}^{t}(t-s)^{\alpha-1} f(s) d s \\
\text { a.e. } t \in[0, b] .
\end{array}
$$

Let $K$ be a compact and convex set in $C\left([0, b], \mathbb{R}^{N}\right)$ such that $S_{c} \subset K$. Given that $y_{*} \in K$ and $\epsilon>0$, we define the following multifunction $U_{\epsilon}:[0, b] \rightarrow \mathscr{P}\left(\mathbb{R}^{N}\right)$ by

$$
\begin{gathered}
U_{\epsilon}(t)=\left\{u \in \mathbb{R}^{N}:\|f(t)-u\|<d(f(t), F(t, y(t)))+\epsilon,\right. \\
\left.u \in F\left(t, y_{*}(t)\right)\right\} .
\end{gathered}
$$


The multivalued map $t \rightarrow F(t, \cdot)$ is measurable and $x \rightarrow$ $F(\cdot, x)$ is $H_{d}$ continuous. In addition, if $F(\cdot, \cdot)$ has compact values, then $F(\cdot, \cdot)$ is graph measurable, and the mapping $t \rightarrow F(t, y(t))$ is a measurable multivalued map for fixed $y \in$ $C\left([0, b], \mathbb{R}^{N}\right)$. Then by Lemma 3 , there exists a measurable selection $v_{1}(t) \in F(t, y(t))$ a.e. $t \in[0, b]$ such that

$$
\left\|f(t)-v_{1}(t)\right\|<d(f(t), F(t, y(t)))+\epsilon ;
$$

this implies that $U_{\epsilon}(\cdot) \neq \emptyset$. We consider $G_{\epsilon}: K \rightarrow \mathscr{P}\left(L^{1}(J\right.$, $\mathbb{R}^{N}$ ) defined by

$$
\begin{array}{r}
G_{\epsilon}(y)=\left\{f_{*} \in \mathscr{F}(y):\left\|f(t)-f_{*}(t)\right\|\right. \\
\left.<\epsilon+d\left(f_{*}(t), F(t, y(t))\right)\right\} .
\end{array}
$$

Since the measurable multifunction $F$ is integrable bounded, Lemma 9 implies that the Nemyts'kil operator $\mathscr{F}$ has decomposable values. Hence $y \rightarrow \overline{G_{\epsilon}(y)}$ is l.s.c. with decomposable values. By Lemma 8 , there exists a continuous selection $f_{\epsilon}$ : $C\left([0, b], \mathbb{R}^{N}\right) \rightarrow L^{1}\left(J, \mathbb{R}^{N}\right)$ such that

$$
f_{\epsilon}(y) \in \overline{G_{\epsilon}(y)} \quad \forall y \in C\left([0, b], \mathbb{R}^{N}\right) .
$$

From Theorem 17, there exists function $g_{\epsilon}: K \rightarrow L_{w}([0, b]$, $\mathbb{R}^{N}$ ) such that

$$
\begin{gathered}
g_{\epsilon}(y) \in \operatorname{ext} S_{F}(y)=S_{\operatorname{ext} F(\cdot, y(\cdot))} \quad \forall y \in K, \\
\left\|g_{\epsilon}(y)-f_{\epsilon}(y)\right\|_{w} \leq \epsilon, \quad \forall y \in K .
\end{gathered}
$$

From $\left(\mathscr{H}_{3}\right)$ we can prove that there exists $M>0$ such that

$$
\|y\|_{\infty} \leq M \quad \forall y \in S_{c} .
$$

Consider the sequence $\epsilon_{n} \rightarrow 0$, as $n \rightarrow \infty$, and set $g_{n}=g_{\epsilon_{n}}$, $f_{n}=f_{\epsilon_{n}}$. Set

$$
\begin{array}{r}
V=\left\{f \in L^{1}\left([0, b], \mathbb{R}^{N}\right):\|f(t)\| \leq \psi(t) \text { a.e. } t \in[0, b]\right\}, \\
\psi(t)=(1+M) p(t) .
\end{array}
$$

Let $L: V \rightarrow C\left([0, b], \mathbb{R}^{N}\right)$ be the map such that each $f \in V$ assigns the unique solution of the problem

$$
\begin{aligned}
& { }^{c} D^{\alpha} y(t)=f(t), \quad \text { a.e. } t \in[0, b], \\
& y(0)=y_{0}, \quad y^{\prime}(0)=y_{1} .
\end{aligned}
$$

As in Theorem 24, we can prove that $L(V)$ is compact in $C\left([0, b], \mathbb{R}^{N}\right)$ and the operator $N_{n}=L \circ g_{n}: K \rightarrow K$ is compact; then by Schauder's fixed point there exists $\tilde{y}_{n} \in K$ such that $\tilde{y}_{n} \in S_{e}$ and

$$
\begin{array}{r}
\widetilde{y}_{n}(t)=y_{0}+t y_{1}+\frac{1}{\Gamma(\alpha)} \int_{0}^{t}(t-s)^{\alpha-1} g_{n}\left(y_{n}\right)(s) d s, \\
\text { a.e. } t \in[0, b], n \in \mathbb{N} .
\end{array}
$$

Hence

$$
\begin{aligned}
\left\|y(t)-\tilde{y}_{n}(t)\right\| & \\
\leq & \frac{1}{\Gamma(\alpha)}\left\|\int_{0}^{t}(t-s)^{\alpha-1}\left[g_{n}\left(\tilde{y}_{n}\right)(s)-f(s)\right] d s\right\| \\
\leq & \frac{1}{\Gamma(\alpha)}\left\|\int_{0}^{t}(t-s)^{\alpha-1}\left[g_{n}\left(\tilde{y}_{n}\right)(s)-f_{n}\left(\tilde{y}_{n}\right)(s)\right] d s\right\| \\
& +\frac{b^{\alpha}}{\Gamma(\alpha)} \int_{0}^{t}\left\|f_{n}\left(\tilde{y}_{n}\right)(s)-f(s)\right\| d s \\
\leq & \frac{1}{\Gamma(\alpha)}\left\|\int_{0}^{t}(t-s)^{\alpha-1}\left[g_{n}\left(\tilde{y}_{n}\right)(s)-f_{n}\left(\tilde{y}_{n}\right)(s)\right] d s\right\| \\
& +\frac{b^{\alpha}}{\Gamma(\alpha)} \int_{0}^{t}\left(\epsilon_{n}+d\left(f(s), f_{n}\left(\tilde{y}_{n}\right)(s)\right)\right) d s \\
\leq & \frac{1}{\Gamma(\alpha)}\left\|\int_{0}^{t}(t-s)^{\alpha-1}\left[g_{n}\left(\tilde{y}_{n}\right)(s)-f_{n}\left(\tilde{y}_{n}\right)(s)\right] d s\right\| \\
& +\frac{b^{\alpha}}{\Gamma(\alpha)} \int_{0}^{t}\left(\epsilon_{n}+H_{d}\left(F(s, y(s)), F\left(s, \tilde{y}_{n}(s)\right)\right)\right) d s \\
\leq & \frac{b^{\alpha+1}}{\Gamma(\alpha+1)} \epsilon_{n}+\frac{b^{\alpha+1}}{\Gamma(\alpha)} \epsilon_{n}+\int_{0}^{t} p(s)\left\|y(s)-\tilde{y}_{n}(s)\right\| .
\end{aligned}
$$

Let $\tilde{y}(\cdot)$ be a limit point of the sequence $\tilde{y}_{n}(\cdot)$. Then, it follows that from the above inequality, one has

$$
\|y(t)-\tilde{y}(t)\| \leq \int_{0}^{t} p(s)\|y(s)-\tilde{y}(s)\| d s
$$

which implies $y(\cdot)=\tilde{y}(\cdot)$. Consequently, $y \in S_{c}$ is a unique limit point of $\tilde{y}_{n}(\cdot) \in S_{e}$.

Example 26. Let $F: J \times \mathbb{R}^{N} \rightarrow \mathscr{P}_{\mathrm{cpcv}}\left(\mathbb{R}^{N}\right)$ with

$$
F(t, y)=\bar{B}\left(f_{1}(t, y), f_{2}(t, y)\right)
$$

where $f_{1}, f_{2}: J \times \mathbb{R}^{N} \rightarrow \mathbb{R}^{N}$ are Carathéodory functions and bounded.

Then (2) is solvable.

Example 27. If, in addition to the conditions on $F$ of Example 26, $f_{1}$ and $f_{2}$ are Lipschitz functions, then $\overline{S_{e}}=S_{c}$.

\section{Acknowledgments}

This work is partially supported by the Ministerio de Economia y Competitividad, Spain, project MTM2010-15314, and cofinanced by the European Community Fund FEDER. 


\section{References}

[1] K. Diethelm and A. D. Freed, "On the solution of nonlinear fractional order differential equations used in the modeling of viscoplasticity," in Scientifice Computing in Chemical Engineering II-Computational Fluid Dynamics, Reaction Engineering and Molecular Properties, F. Keil, W. Mackens, H. Voss, and J. Werther, Eds., pp. 217-224, Springer, Heidelberg, Germany, 1999.

[2] L. Gaul, P. Klein, and S. Kemple, "Damping description involving fractional operators," Mechanical Systems and Signal Processing, vol. 5, no. 2, pp. 81-88, 1991.

[3] W. G. Glockle and T. F. Nonnenmacher, "A fractional calculus approach to self-similar protein dynamics," Biophysical Journal, vol. 68, no. 1, pp. 46-53, 1995.

[4] F. Mainardi, "Fractional calculus: some basic problems in continuum and statistical mechanics," in Fractals and Fractional Calculus in Continuum Mechanics, A. Carpinteri and F. Mainardi, Eds., pp. 291-348, Springer, Wien, Germany, 1997.

[5] A. B. Malinowska and D. F. M. Torres, "Towards a combined fractional mechanics and quantization," Fractional Calculus and Applied Analysis, vol. 15, no. 3, pp. 407-417, 2012.

[6] R. Metzler, W. Schick, H. Kilian, and T. F. Nonnenmacher, "Relaxation in filled polymers: a fractional calculus approach," The Journal of Chemical Physics, vol. 103, no. 16, pp. 7180-7186, 1995.

[7] S. P. Näsholm and S. Holm, "On a fractional Zener elastic wave equation," Fractional Calculus and Applied Analysis, vol. 16, no. 1, pp. 26-50, 2013.

[8] L. Vázquez, J. J. Trujillo, and M. P. Velasco, "Fractional heat equation and the second law of thermodynamics," Fractional Calculus and Applied Analysis, vol. 14, no. 3, pp. 334-342, 2011.

[9] B. J. West and D. West, "Fractional dynamics of allometry," Fractional Calculus and Applied Analysis, vol. 15, no. 1, pp. 7096, 2012.

[10] A. A. Kilbas, H. M. Srivastava, and J. J. Trujillo, Theory and Applications of Fractional Differential Equations, vol. 204 of North-Holland Mathematics Studies, Elsevier Science B.V., Amsterdam, The Netherland, 2006.

[11] K. S. Miller and B. Ross, An Introduction to the Fractional Calculus and Differential Equations, John Wiley, New York, NY, USA, 1993.

[12] I. Podlubny, Fractional Differential Equations, Academic Press, San Diego, Calif, USA, 1999.

[13] S. G. Samko, A. A. Kilbas, and O. I. Marichev, Fractional Integrals and Derivatives, Theory and Applications, Gordon and Breach Science, Yverdon, Switzerland, 1993.

[14] A. A. Kilbas and J. J. Trujillo, "Differential equations of fractional order: methods, results and problems. II," Applicable Analysis, vol. 81, no. 2, pp. 435-493, 2002.

[15] A. M. Nahušev, "The Sturm-Liouville problem for a second order ordinary differential equation with fractional derivatives in the lower terms," Doklady Akademii Nauk SSSR, vol. 234, no. 2, pp. 308-311, 1977.

[16] I. Podlubny, I. Petráš, B. M. Vinagre, P. O’Leary, and L. Dorčák, "Analogue realizations of fractional-order controllers," Nonlinear Dynamics, vol. 29, no. 1-4, pp. 281-296, 2002.

[17] C. Yu and G. Gao, "Existence of fractional differential equations," Journal of Mathematical Analysis and Applications, vol. 310, no. 1, pp. 26-29, 2005.
[18] V. Lakshmikantham, "Theory of fractional functional differential equations," Nonlinear Analysis: Theory, Methods \& Applications A, vol. 69, no. 10, pp. 3337-3343, 2008.

[19] Y. Chalco-Cano, J. J. Nieto, A. Ouahab, and H. Román-Flores, "Solution set for fractional differential equations with RiemannLiouville derivative," Fractional Calculus and Applied Analysis, vol. 16, no. 3, pp. 682-694, 2013.

[20] J. T. Machado, V. Kiryakova, and F. Mainardi, "Recent history of fractional calculus," Communications in Nonlinear Science and Numerical Simulation, vol. 16, no. 3, pp. 1140-1153, 2011.

[21] S. Abbas and M. Benchohra, "Fractional order RiemannLiouville integral inclusions with two independent variables and multiple delay," Opuscula Mathematica, vol. 33, no. 2, pp. 209-222, 2013.

[22] S. Abbas, M. Benchohra, and J. J. Nieto, "Global uniqueness results for fractional order partial hyperbolic functional differential equations," Advances in Difference Equations, vol. 2011, Article ID 379876, 25 pages, 2011.

[23] M. Benchohra, J. Henderson, S. K. Ntouyas, and A. Ouahab, "Existence results for fractional functional differential inclusions with infinite delay and applications to control theory," Fractional Calculus \& Applied Analysis, vol. 11, no. 1, pp. 35-56, 2008.

[24] J. Henderson and A. Ouahab, "Fractional functional differential inclusions with finite delay," Nonlinear Analysis: Theory, Methods \& Applications A, vol. 70, no. 5, pp. 2091-2105, 2009.

[25] J. Henderson and A. Ouahab, "Impulsive differential inclusions with fractional order," Computers \& Mathematics with Applications, vol. 59, no. 3, pp. 1191-1226, 2010.

[26] F. Jiao and Y. Zhou, "Existence of solutions for a class of fractional boundary value problems via critical point theory," Computers \& Mathematics with Applications, vol. 62, no. 3, pp. 1181-1199, 2011.

[27] A. Ouahab, "Some results for fractional boundary value problem of differential inclusions," Nonlinear Analysis: Theory, Methods \& Applications A, vol. 69, no. 11, pp. 3877-3896, 2008.

[28] A. Ouahab, "Filippov's theorem for impulsive differential inclusions with fractional order," Electronic Journal of Qualitative Theory of Differential Equations, no. 23, pp. 1-23, 2009.

[29] A. Ouahab, "Fractional semilinear differential inclusions," Computers \& Mathematics with Applications, vol. 64, no. 10, pp. 3235-3252, 2012.

[30] F. S. de Blasi and G. Pianigiani, "Non-convex valued differential inclusions in Banach spaces," Journal of Mathematical Analysis and Applications, vol. 128, pp. 541-555, 1996.

[31] N. S. Papageorgiou, "On the "bang-bang" principle for nonlinear evolution inclusions," Aequationes Mathematicae, vol. 45, no. 2-3, pp. 267-280, 1993.

[32] A. Tolstonogov, Differential Inclusions in a Banach Space, Kluwer Academic, Dordrecht, The Netherlands, 2000.

[33] A. A. Tolstonogov, "Extreme continuous selectors of multivalued maps and their applications," Journal of Differential Equations, vol. 122, no. 2, pp. 161-180, 1995.

[34] A. A. Tolstonogov, "Extremal selectors of multivalued mappings and the, "bangbang" principle for evolution inclusions," Doklady Akademii Nauk SSSR, vol. 317, no. 3, pp. 589-593, 1991 (Russian), translation in Soviet Mathematics Doklady, vol. 43, no. 2, 481-485, 1991.

[35] J.-P. Aubin and A. Cellina, Differential Inclusions, Springer, Berlin, Germany, 1984. 
[36] J.-P. Aubin and H. Frankowska, Set-Valued Analysis, Birkhäuser, Boston, Mass, USA, 1990.

[37] S. Hu and N. S. Papageorgiou, Handbook of Multivalued Analysis, Volume I: Theory, Kluwer Academic Publishers, London, UK, 1997.

[38] M. Kisielewicz, Differential Inclusions and Optimal Control, Kluwer Academic, Dordrecht, The Netherlands, 1991.

[39] Q. J. Zhu, "On the solution set of differential inclusions in Banach space," Journal of Differential Equations, vol. 93, no. 2, pp. 213-237, 1991.

[40] A. Bressan and G. Colombo, "Extensions and selections of maps with decomposable values," Studia Mathematica, vol. 90, no. 1, pp. 70-85, 1988.

[41] M. Frigon and A. Granas, "Théorèmes d'existence pour des inclusions différentielles sans convexité," Comptes Rendus de l'Académie des Sciences. Série I, vol. 310, no. 12, pp. 819-822, 1990.

[42] N. Dunford and J. T. Schwartz, Linear Operators. I. General Theory, With the Assistance of W. G. Bade and R. G. Bartle, Pure and Applied Mathematics, Interscience Publishers, New York, NY, USA, 1958.

[43] R. D. Bourgin, Geometric Aspects of Convex Sets with the RadonNikody'm Property, vol. 993 of Lecture Notes in Mathematics, Springer, Berlin, Germany, 1983.

[44] M. Florenzano and C. Le Van, Finite Dimensional Convexity and Optimization, vol. 13 of Studies in Economic Theory, Springer, Berlin, Germany, 2001, In Cooperation with Pascal Gourdel.

[45] S. Abbas, M. Benchohra, and G. M. N’Guérékata, Topics in Fractional Differential Equations, vol. 27 of Developments in Mathematics, Springer, New York, NY, USA, 2012.

[46] M. Caputo, Elasticita e Dissipazione, Zanichelli, Bologna, Italy, 1969.

[47] M. Caputo, "Linear models of dissipation whose Q is almost frequency independent, part II," Geophysical Journal of the Royal Astronomical Society, vol. 13, pp. 529-529, 1967.

[48] M. Caputo and F. Mainardi, "Linear models of dissipation in anelastic solids," La Rivista del Nuovo Cimento, vol. 1, no. 2, pp. 161-198, 1971.

[49] M. Benamara, Point extremaux, multi-applications et fonctionnelles integrales [Thése de 3éme Cycle], Universite de Grenoble, Grenoble, France, 1975.

[50] F. S. de Blasi and G. Pianigiani, "Baire's category and the bangbang property for evolution differential inclusions of contractive type," Journal of Mathematical Analysis and Applications, vol. 367, no. 2, pp. 550-567, 2010.

[51] B. Ahmad and J. J. Nieto, "A study of impulsive fractional differential inclusions with anti-periodic boundary conditions," Fractional Differential Calculus, vol. 2, no. 1, pp. 1-15, 2012.

[52] B. Ahmad and J. J. Nieto, "Existence results for higher order fractional differential inclusions with nonlocal boundary conditions," Nonlinear Studies, vol. 17, no. 2, pp. 131-138, 2010. 


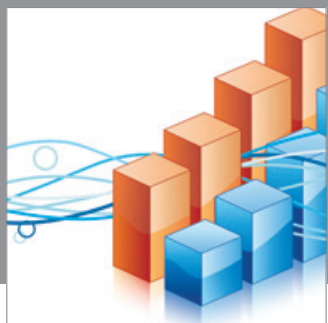

Advances in

Operations Research

mansans

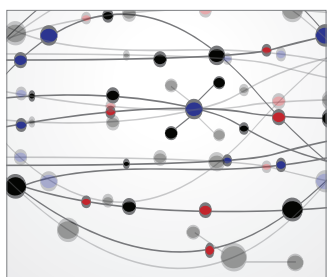

The Scientific World Journal
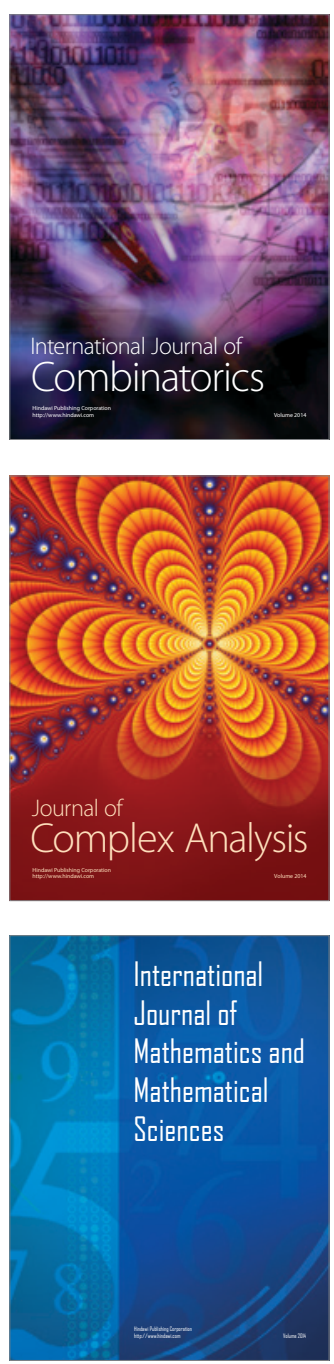
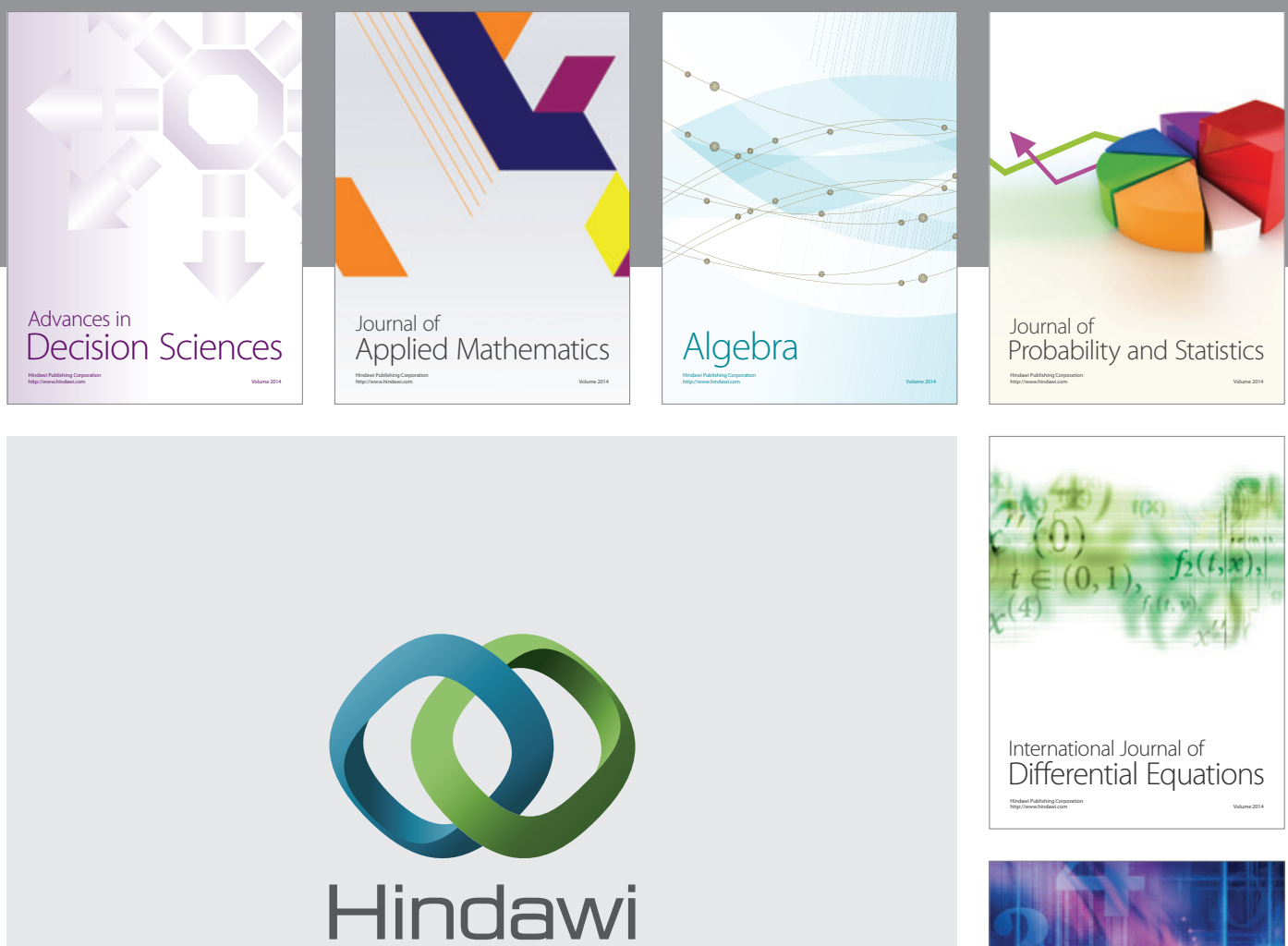

Submit your manuscripts at http://www.hindawi.com
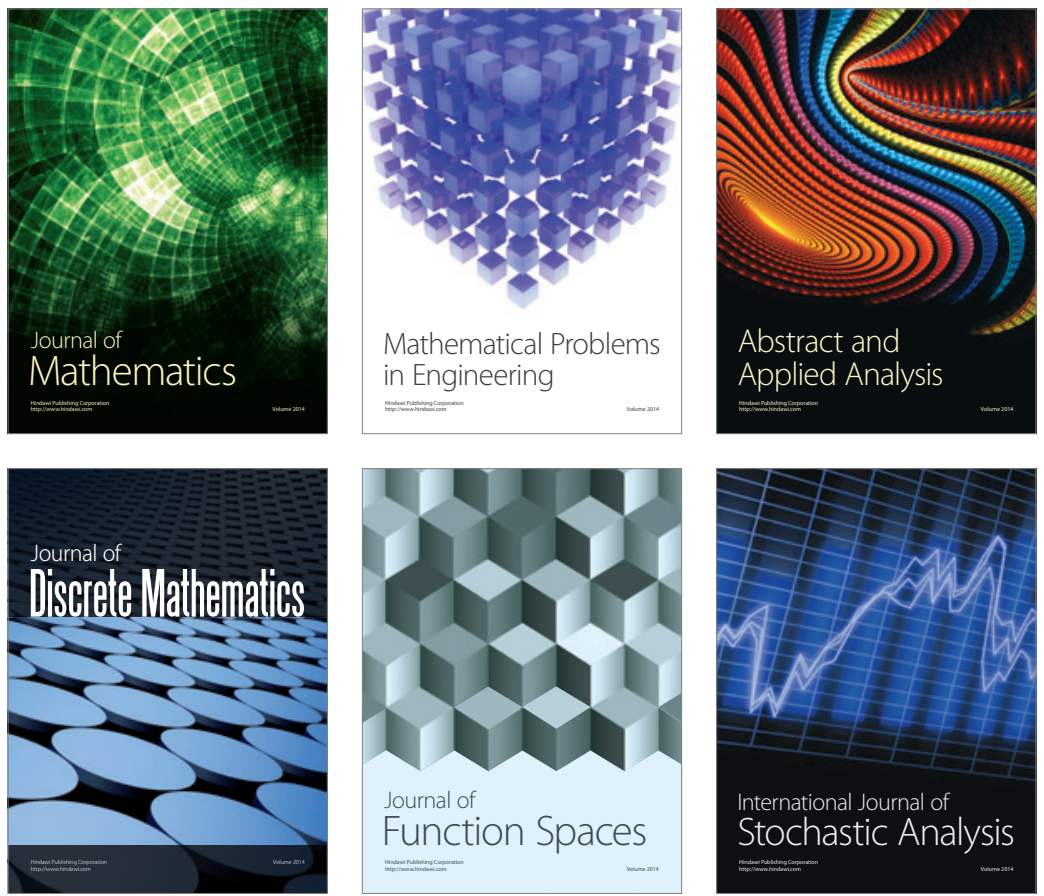

Journal of

Function Spaces

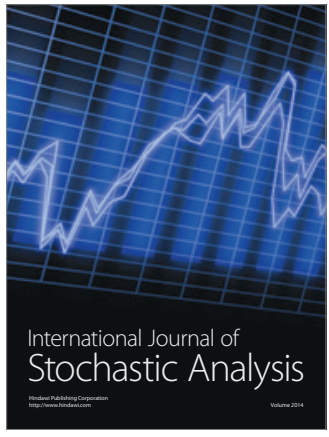

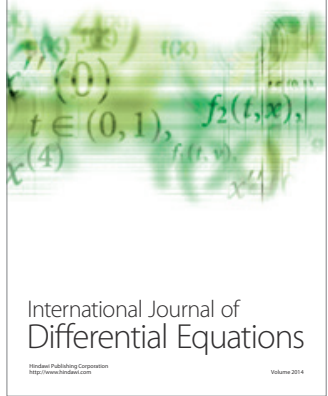
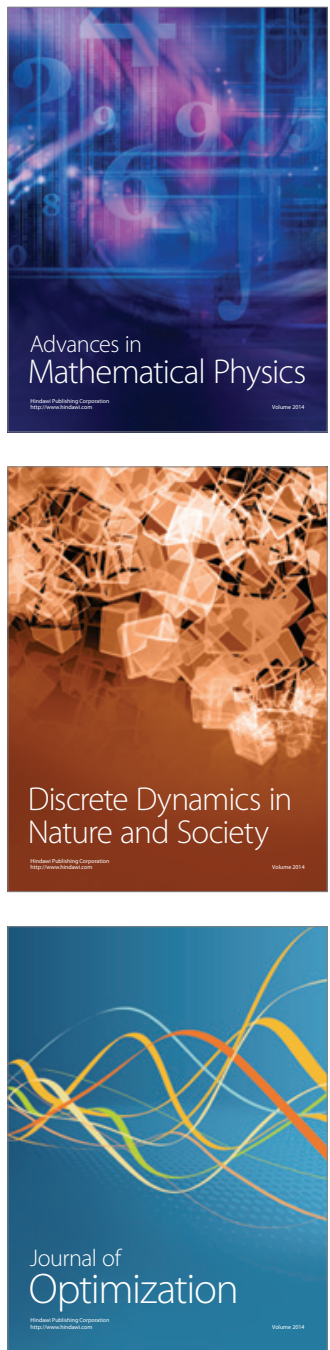